\title{
TURISMO Y VIDA COTIDIANA: UN ENFOQUE COMPLEJO DESDE ESPACIO-TIEMPO
}

\author{
Rossana Campodónico* y Luis Chalar ${ }^{* *}$ \\ Universidad de la República (Uruguay)
}

\section{RESUMEN}

El presente ensayo profundiza en el abordaje del continuo distanciamiento/acercamiento entre vida cotidiana (VC) y turismo, componentes importantes de la teoría turística, haciendo hincapié en la centralidad de la dimensión espaciotemporal. En concreto se intentará responder a la interrogante ¿qué características presenta la triada $\mathrm{VC} /$ turismo/movilidad desde la perspectiva del binomio espacio-tiempo y qué puede implicar en el turismo? Entre los resultados obtenidos se destaca que las variables de amplio rango se potencian en su combinación y más aún si se entrecruzan con valores diferentes a nivel de individuos, grupos de actores, así como para los productos y destinos turísticos.

Palabras Clave: turismo, vida cotidiana, movilidad, espacio, tiempo.

\section{TOURISM AND DAILY LIFE: A COMPLEX APPROACH FROM THE SPACE-TIME}

\section{Abstract}

This essay delves into the approach of the continuous distancing/rapprochement between daily life and tourism, important components of tourism theory, emphasizing the spacetime dimension, since it is understood that, given its centrality, they deserve further reflection. Specifically, an attempt will be made to answer the question: what characteristics does the $\mathrm{VC} /$ tourism / mobility trilogy present from the perspective of the space-time binomial and what can it imply in tourism? Among the results obtained, it is highlighted that the wide-range variables are enhanced in their combination and even more so if they intersect with different values at the level of individuals, groups of actors, as well as for tourism products and destinations.

KEYwORDs: tourism, daily life, mobility, space, time. 


\section{INTRODUCCIÓN}

Este ensayo pretende profundizar sobre el continuo distanciamiento/acercamiento entre vida cotidiana y turismo, partiendo de la necesidad surgida en un artículo realizado por R. Campodónico y L. Chalar (2019), de profundizar sobre el componente espaciotemporal del Campo de Análisis TEMA (Tiempo, Espacio, Motivaciones y Actividades) al momento de indagarse sobre la triada vida cotidiana (VC), turismo y movilidad.

Asimismo, se entendió como relevante atender lo expresado por A. Giddens (1984, en Hall, 2009) cuando, enmarcando a los patrones colectivos como la suma de trayectos y conductas individuales, sostiene que "Los científicos sociales, incluidos muchos de los que analizan el turismo, no han logrado estructurar sus razonamientos alrededor de los modos en que los sistemas sociales se constituyen a través del espacio-tiempo» (p. 105).

Definido este punto de partida, se tuvo como objetivo dar respuesta a la interrogante ¿̇cómo se presenta la relación entre los conceptos abordados (VC/turismo/ movilidad) mediados por la dimensión espaciotemporal y qué puede implicar en el turismo?

Esta reflexión teórica busca abordar conceptualmente como VC, turismo y movilidad (esta última como conector entre las primeras), son definidos, en gran medida, desde lo espaciotemporal y se reconfiguran en base a ello. Por tal motivo se recurre a un conjunto de autores de diversas disciplinas, como así también a casos prácticos, lo que permite reflexionar para integrar parte de la teoría existente, contribuir a su avance y facilitar su comprensión.

\section{MARCO CONCEPTUAL}

LA DIMENSIÓN ESPACIOTEMPORAL

Los individuos, a través de la vida, se ubican en determinadas dimensiones espaciotemporales, conceptos que se vuelven centrales en el análisis de la vida cotidiana, el turismo y la movilidad. Ambos son «... los componentes situacionales dentro de la construcción de la realidad turística, aportando sus coordenadas espacio-temporales y conformando la base histórica y física del proceso" (Campodónico y Chalar, 2014, p. 13). En el mismo sentido, M. Hall (2009) al analizar un conjunto de definiciones de turismo, resalta como de especial importancia en ellas los conceptos de «espacio" (alejarse de la zona de origen) «... y "tiempo" (cantidad de tiempo fuera de su zona de origen)» (p. 39).

*E-mail:campodon@gmail.com.

**E-mail: chalarluis@gmail.com. 
El desplazamiento resultante del viaje permite vivenciar un lugar y un tiempo que desde la visión del sujeto (turista) puede ser la contracara de la vida cotidiana, como lo refleja la postura sostenida por J. Urry (2004): «El turismo surge de una división binaria fundamental entre lo ordinario/cotidiano y lo extraordinario. Las experiencias turísticas implican algún elemento o aspecto que induce experiencias placenteras que, en comparación con lo cotidiano salen fuera de lo común» (pp. 16-17).

Asimismo, el individuo, al igual que la sociedad que conforma, tiene una trayectoria histórica determinada donde prima de manera absoluta la vida cotidiana por ser la más extensa en el tiempo, esa que ocurre todos los días aún sin ser exactamente igual. Lo que se complementa en la expresión de A. Heller (1998) cuando dice: «... la vida cotidiana pareciera ser homogénea para todos los miembros de una misma comunidad; sin embargo, cuando se la examina de cerca, es apreciable que ella tiene poco en común entre las mismas personas» (p. 37).

Una definición clásica como la de E. Pichón Riviére y A. Pampliega (1985) hace referencia a la VC como «... el espacio y el tiempo en que se manifiestan, en forma inmediata, las relaciones que los hombres establecen entre sí y con la naturaleza en función de sus necesidades» (p. 12).

Pero también se considera lo sostenido por A. Mantecón (2008) sobre las características actuales de la investigación sociológica del ocio y que caracteriza a la VC, cuando refiere que si bien «... la moderna y creciente tendencia a segmentar el tiempo cotidiano en compartimientos independientes. Las sociedades actuales se vuelven más complejas y las dimensiones, indicadores y variables que explican el ocio se tornan difusos» (p. 46).

Si se tienen en cuenta las tres dimensiones que actúan en lo cotidiano (tiempo, espacio e interdependencia) en opinión de J. Gerlero y D. Taranda (2005), estas tendrán su influencia en el individuo cuando hace turismo y lo expresan del siguiente modo:

... a partir del estudio de la vida cotidiana, como mundo de lo heterogéneo, de lo racional y a la vez de lo emocional, de lo diverso, pero a la vez de lo rutinario, es posible acceder a la explicación y análisis de las prácticas de los sujetos en diversos espacios y temporalidades, por ejemplo, las vinculadas a la recreación y el turismo, que aparecen a la mirada de una observación ingenua como escindidas de la misma (p. 141).

En este punto parece interesante contrastar lo anterior con las ideas de A. Lindón (2012) cuando sostiene que

Las teorías sobre la vida cotidiana casi siempre reconocen que una de sus grandes dilemas es si la vida cotidiana está dominada por la rutinización, lo repetitivo y en consecuencia, es el campo de la reproducción social e incluso, de la alienación y la colonización del sujeto. O bien, si la vida cotidiana es fuente de invención y creatividad, y por lo mismo lo cotidiano podría contribuir a la producción o a la trasformación social (en Lindón y Hiernaux, 2012, p. 74).

La dimensión espaciotemporal, entonces, se encuentra en la vida cotidiana al igual que la práctica turística, la que tiene un valor temporal menor que la citada 
anteriormente, aunque se distingue por tener una carga simbólica significativa que contribuye a la formación de los imaginarios sociales. Cabe acotar que el espacio que se estudia es inacabado por definición, está en un estado de transformación permanente y es apreciado por el individuo solo en el tiempo y por su intermedio, al decir de T. Unwin (2000).

Complementariamente, la forma de comprender la trayectoria de los individuos - como de los destinos turísticos- es cuando se analiza la movilidad en un sentido amplio, concepto clave para explicar el fenómeno turístico. Dicha movilidad tiene varias dimensiones y eso se aprecia en la definición dada por K. Hannam et al. (2006, en Onghena y Milano, 2015) cuando hacen referencia a que es lo que se mueve (personas, objetos, capital e información) y el ámbito donde lo hace (global-local; vida cotidiana).

De ahí la importancia de abordar el turismo como una movilidad específica, que en opinión de L. Collado Medina et al. (2013) debe ser entendida como

La movilidad turística será uno de los factores que reconstituyan la vida social de manera compleja y desigual en el proceso de globalización, a partir del significado de los objetos, los sentidos, el tiempo y el espacio. Dicho cambio deberá ser estudiado en su diversidad contextual, porque al enfrentarnos a un mundo globalizado, los patrones culturales y los estilos de vida social presentarán procesos constantes de transformación (p. 29).

En este sentido, M. Hall (2009) pone de manifiesto perspectivas cuantitativas y cualitativas cuando sostiene que «... parece que el turismo está siendo interpretado cada vez más como una sola -aunque tremendamente significativa- dimensión de la movilidad y la circulación pasajeras» (p. 45), a la vez, que argumenta que «La cifra de desplazamientos desciende más a medida que los viajes son hasta lugares más lejanos temporal y espacialmente del punto de origen» (p. 45).

\section{LA DIMENSIÓN ESPACIAL}

En cuanto al concepto de espacio existen múltiples definiciones, pero a los efectos de este trabajo se contempla la de M. Santos (1994) cuando sostiene que se trata de «... sistemas de acciones y sistemas de objetos indisolublemente unidos y cuya suma e interacción nos dan el espacio total» (pp. 48-49), destacándose que tiene «... una gran cantidad y diversidad de objetos, esto se forma por el número exponencial de acciones, es decir, flujos, que lo cruzan» (p. 17) ${ }^{1}$.

Será en esos espacios donde se producirá la máxima expresión de la ambivalencia de lo cotidiano / no cotidiano, pues representa lo primero para los anfitriones/ comunidad que recibe a los turistas y donde tienen cabida la oferta de bienes y servicios. La mayoría de los abordajes sobre la dimensión espacial se realizan desde la

\footnotetext{
1 Traducción de los autores.
} 
perspectiva del turista (sujeto), relegando otros actores del sistema turístico. Desde la posición del turista (sujeto), el espacio del destino seleccionado es distinto del habitual, por lo que esa característica de «nuevo» implica que tiene una significación de lo extraordinario, incluso en aquellos casos donde el sujeto haya ya estado en otras oportunidades (repetición de la visita).

Si se posiciona desde la VC, el espacio es el habitual para los residentes de un destino o lugar turístico, lo que implica que tienen un uso amplio e ilimitado del mismo, donde se transita por la multiplicidad de los diferentes espacios contenidos en él. El residente tiene una apropiación de ese espacio por ser el suyo, su aquí (lo común y regular), y le confiere identidad, o mejor dicho, la determina, no solo por su propia experiencia en él sino también por la trayectoria histórica que ha recibido como legado y que es el sustento de la historicidad de la VC.

Complementariamente, se comparte lo sostenido por A. Lindón (en Lindón y Hiernaux, 2012), cuando manifiesta que no se puede entender que el espacio se reduzca a lo estrictamente material, sino que se requiere de perspectivas más amplias que lo desborden. De acuerdo con esta argumentación, si bien en un determinado espacio se producen diferentes comportamientos, estos dependen de «... una tercera esfera, la del significado, cuya autonomía y papel mediador deben mucho al imaginario» (Berdoulay, 2012 en Lindón y Hiernaux, 2012, p. 61). En este sentido, se adhiere a lo expresado por A. Lindón (2012) cuando dice que «En toda experiencia espacial se incluyen los significados y sentidos que le otorgamos a nuestros espacios de la vida» (en Lindón y Hiernaux, 2012, p. 70).

Se alude a un proceso de coconstrucción del sujeto y del lugar por lo que «... el imaginario es movilizado, modificado, trabajado una y otra vez, de tal suerte que se llega a otorgar nuevos sentidos a las acciones» (Lindón y Hiernaux, 2012, p. 52). Pero tratando de trascender lo meramente físico del espacio, se retoma lo planteado por V. Berdoulay (2012), quien sostiene que «El sujeto se construye a sí mismo y significa su compromiso en el mundo, contribuyendo así a redefinir el lugar en el cual se inserta» (en Lindón y Hiernaux, 2012, p. 50).

También debe considerarse lo que el autor denomina «escenificación», es decir, la puesta en escena del espacio público por parte de la administración que ordena un determinado territorio donde se produce una «... reducción narrativa, de lo que nos hace ver la vida cotidiana» (Berdoulay, 2012 en Lindón y Hiernaux, 2012, p. 62).

A lo cual debe agregarse que existen determinados lugares que son adecuados o readecuados para las actividades turísticas por lo que existen políticas y/o acciones tomadas por las administraciones públicas -y la mayoría de las veces con inversiones de actores privados- que irán a producir modificaciones sustanciales en algunos casos en ese espacio que es el habitual de la comunidad. Esto implica que parte de la infraestructura de productos y servicios desarrollados con fines turísticos son utilizados por el residente mediante un uso residual del mismo y que también es resignificado desde la conceptualización del ocio y del tiempo libre.

Por otra parte, R. Campodónico y L. Chalar (2014) han expresado que el espacio tiene como principal característica ser «multidimensional», en virtud de lo cual sería mejor hablar de "espacios" ya que en un destino/lugar turístico las movilidades y los flujos que se dan en él son múltiples y generan distintas situaciones. 
La noción de tiempo, construcción subjetiva y con distintos grados de percepción, le aporta al individuo un sentido relacional y de historicidad en el mundo cotidiano y en el no cotidiano.

En el campo del turismo, el tiempo se puede apreciar en distintos niveles ya que dependerá del objeto en sí como de su historia y el grado de desarrollo del destino, lugar, zona, región y/o país, así como su trayectoria en el devenir histórico. A la vez que, desde la representación del sujeto (turista/no turista), la valoración del tiempo es distinta y nunca presenta una percepción uniforme (Campodónico y Chalar, 2014).

En lo que refiere al tiempo histórico, que es lo que define al sujeto a lo largo de su vida, se parte de lo planteado por R. Koselleck (1993) cuando sostiene que

El pasado y el futuro no han llegado a coincidir nunca, como tampoco se puede deducir totalmente una expectativa a partir de la experiencia. Una vez reunida, una experiencia es tan completa como pasados son sus motivos, mientras que la experiencia futura, lo que va a hacer, anticipada como expectativa se descompone en una infinidad de trayectos temporales diferentes (p. 339).

En el caso del turismo, esta conceptualización es fundamental para comprender el proceso en el cual el individuo manifiesta distintos comportamientos a lo largo de su trayectoria. Por eso el autor sostiene que la experiencia-que proviene del pasado- tiene un sustrato espacial que, a su vez, se vincula fuertemente con diferentes etapas de tiempos anteriores. En turismo, el pasado y el futuro son parte de la VC del sujeto pues el acto en sí de viajar se manifiesta en tiempo presente y no cotidiano.

El viaje es un largo proceso que ha sido definido por la mayoría de los autores en tres etapas (antes, durante y después del viaje). Por lo que comienza a ser planeado en su VC (antes), lo vive como presente efímero en el destino mismo (espacio no cotidiano) y lo vuelve a revivir en su VC (espacio habitual) en el llamado "después del viaje» mediante los relatos, comentarios, fotografías, suvenires, entre otros.

Si bien el punto de inflexión más importante es cuando el viaje se plasma en la realidad turística, cuando las expectativas de las motivaciones pueden ser contrastadas con las actividades realizadas en el destino seleccionado. Esta constatación se dará por medio del disfrute, que adquiere valores simbólicos importantes que son retomados desde el «antes» pues allí se encuentran las motivaciones esenciales que lo llevaron a moverse a un espacio diferente.

Las características actuales del turista hacen que los viajes ahora sean más fraccionados a lo largo del año, por lo cual esta secuencia detallada anteriormente ocurre varias veces en ese período, permeando así de modo constante a la VC.

También se comparte la opinión de M. Hall (2009) cuando expresa que «... los estudios sobre los patrones y los flujos de movimiento turísticos a gran escala no deberían perder de vista nunca a la persona que se mueve en el espacio y en el tiempo a título individual» (p. 47). Si bien el individuo puede integrar un grupo de 
viaje, de hecho comparte con un número importante de otros sujetos los medios de transporte que le facilitan el desplazamiento, el viaje es personal, vivido de manera intransferible y percibido como único: es su cuerpo en un espacio distinto al cotidiano y es su tiempo de ocio, que no es igual al de los otros en la significación que adquiere.

Para el residente y los trabajadores del sector, la dimensión temporal refiere a un tiempo habitual que transcurre dentro de los procesos de rutinización laboral, obligaciones familiares y sociales.

\section{ELUCIDACIONES}

\section{Cruce de Dimensiones espaciotemporales}

Si se analiza la relación entre VC, turismo y movilidad se encuentran una variedad de matices que configuran "ventanas» de exploración espaciotemporales. Tanto el tiempo como el espacio presentan tres niveles bien diferenciados, que en el caso del primero implica una mirada hacia el tiempo pasado (antes), una contemplación del presente (ahora) y la atención en el porvenir (futuro).

En el segundo caso, lo concerniente a lo local (aquí), aquello que implica lo local o regional (allí) y los espacios más distantes y distintos (allá). Entre ellas es posible un cruce que permite combinaciones diversas.

\begin{tabular}{ccccc}
\hline \multicolumn{5}{c}{ CUADRO I. CRUCE DE DIMENSIONES ESPACIOTEMPORALES } \\
\hline Antes & AHORA & DesPués \\
\hline Allá & Antes/Allá & Ahora/Allá & Futuro/Allá & Espacial lejano \\
\hline Aquí & Antes/Aquí & Ahora/Aquí & Futuro/Aquí & Espacial local \\
\hline Allí & Antes/Allí & Ahora/Allí & Futuro/Allí & Espacial cercano \\
\hline & Temporal pasado & Temporal actual & Temporal futuro & \\
\hline
\end{tabular}

Fuente: elaboración propia.

En el caso de contemplarse sola una de esas ventanas de intersección se entiende que se está ante un «cuadro» en el entendido de que agrupa a un tiempo y espacio determinado.

Para una fila o columna se entiende que se está ante una «secuencia» de tipo espacial o temporal y de recorrido corto o largo (agrupa dos o tres ventanas respectivamente). Si el espacio/tiempo involucrado abarca dos cuadros pertenecientes a secuencias espaciotemporales diferentes, se está ante un "plano».

Lo enunciado tiene implicancias en tres grandes grupos de actores dentro del turismo, que si bien comparten características se pueden diferenciar para su abordaje. Por una parte: a) aquellos que se encargan de su desarrollo y gestión; b) los que lo investigan y enseñan; y c) quienes lo usufructúan y viven (considerando que una persona puede cumplir distintos roles en etapas diferentes). A continuación, se brindan ejemplos desde un punto de vista general, ilustrándose algunos de ellos con casos concretos y localizados en determinadas zonas de Uruguay. 


\begin{tabular}{|c|c|c|c|c|}
\hline & \multicolumn{4}{|c|}{ CUADRO II. CRUCE DE DIMENSIONES ESPA } \\
\hline & Antes & АноRA & A FUTURO & \\
\hline Allá & $\mathbf{o}$ & $\mathbf{o}$ & $\mathbf{o}$ & ק \\
\hline Aquí & $\mathbf{o}$ & $\mathbf{o}$ & $\mathbf{o}$ & 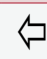 \\
\hline Allí & $\mathbf{o}$ & $\mathbf{o}$ & $\mathbf{o}$ & ح \\
\hline & $\widehat{ن}$ & $\widehat{ن}$ & $\widehat{\imath}$ & \\
\hline
\end{tabular}

Fuente: elaboración propia.

En lo que concierne al grupo (a), que se encargan de su desarrollo y gestión, y dentro de los casos más significativos, se señala a operadores turísticos que incorporan o promueven desde sus ofertas algunas de las principales características de la comunidad del destino/lugar, donde se valora el «aquí» y el «ahora» de la vida cotidiana.

Continuando con el mismo grupo, otra ventana espaciotemporal se centra en el destino, en el "aquí» y el "antes», donde las propuestas pasan por poner en valor construcciones, hechos, relatos sobre lugares donde la historia de la comunidad tiene elementos destacables. Se alude a cuestiones del pasado de la localidad, aquello que sucedió en ese lugar, pero en otro tiempo. Se busca recuperar la historia/memoria de lo que pasó, destacándose tanto lo positivo como lo negativo de la vida cotidiana pasada. Como ejemplos se pueden mencionar las propuestas que se generaron en Uruguay en torno al barrio histórico de Colonia del Sacramento (Departamento de Colonia) y el Frigorífico Anglo (Departamento de Río Negro).

También cabe mencionar la noción de «aquí» en cuanto al espacio y sus posibilidades en el "futuro». En este sentido, se destacan los esfuerzos que se realizan -desde las políticas públicas y/o las iniciativas privadas- por recuperar o reconstruir actividades que están o tienden a desaparecer, que incluyen el desarrollo de posibles futuras alternativas desde una perspectiva innovadora.

Una nueva combinación de las dimensiones de tiempo y espacio transcurre por fuera del «aquí» ya aludido. En ella, la vida cotidiana local no tiene un papel relevante desde el interés de quienes operan en el turismo. En todos los casos, pueden darse diferentes matices que van desde el desconocimiento hasta el desinterés. Esa renuncia de valorar lo local implica dejar un lugar para que se manifiesten otras modalidades con temáticas de origen foráneo, como la común ambientación y animación de los hoteles resorts, donde la vida cotidiana del lugar es diagramada de una manera especial que nada tiene que ver con la de comunidad anfitriona.

Ese recurrir a otros espacios también se puede subdividir de acuerdo a la dimensión temporal mientras que lo que refiere a otras regiones puede implicar una mirada al pasado de las mismas.

Se señalan casos cada vez más recurrentes y relacionados con las estrategias de supervivencia de sectores centrales del turismo, como son los alojamientos y emprendimientos gastronómicos. En el caso de Montevideo - y ante la baja demanda en el sector hotelero- varios establecimientos recurren a prestar nuevos y mayores 
servicios a la comunidad. Entre esas variantes se encuentran brindar servicios para eventos sociales (casamientos, cumpleaños de 15, entre otros); espacios de co-work; eventos culturales (festivales gastronómicos, desfiles de moda, espectáculos musicales, etc.) (diario El País, 2019).

En cuanto al grupo (b), refiere a los que lo investigan y enseñan; merecen un destaque los estudios de casos, ya que estos suelen abrir una ventana espaciotemporal de análisis, lo que permite acceder a conocimientos sobre ese recorte determinado, condición necesaria en ese tipo de investigación. En muchas temáticas la proliferación de casos muy puntuales configura un cúmulo de información que no tiene mayor trascendencia, dada la falta de esfuerzos integradores y de abstracciones que proporcionen cuerpo a la teoría.

Aun en la gran diversidad de situaciones y lo cambiante de las mismas, no parece oportuno dejar de lado el esfuerzo por generar conocimiento en base a contemplar espacios y tiempos más amplios.

Un recorte espaciotemporal limitado permite conocer esa realidad en particular, pero no cuenta con las "credenciales" por sí sola para proyectarse más allá de esa práctica. Esfuerzos a nivel de posgrados, de proyectos interdisciplinarios y/o de líneas de investigación permitirían ir recomponiendo un puzle sobre determinada temática.

Un ejemplo de ello es el caso de estudio de la articulación productiva intersectorial entre sectores productivos (Chalar, 2020), donde se establece como base el estudio una localidad determinada (dimensión espacial), recopilando experiencias de conjunción turismo-agro en diferentes momentos (secuencia temporal). Esa búsqueda necesitaba un recorrido en el espacio mayor, por lo cual se recurrió a la búsqueda de experiencias en la escala regional/nacional e internacional (secuencia espacial). Dicha elección determinó un "plano» de abordaje más general.

Por otra parte, el tener en cuenta a quienes lo usufructúan (grupo c), básicamente el turista/viajero, lleva a preguntarse ¡cuánto de la vida cotidiana del lugar o del visitante está o debe estar presente en el viaje?, ¿cuáles son las proporciones? Sin duda las variantes son muchas y ello ha llevado a realizar menciones que actúan como norte y marcan lo antagónico que pueden ser, cuando la promoción se centra en que el turista debe «sentirse en casa» o por el contrario «si huésped se siente como en su casa, hice todo mal» (diario El Cronista, 2019).

Cuando el turista se desplaza a un destino atraviesa múltiples espacios, primero saliendo de su región de origen, pasando por una zona de tránsito -que puede ser variada dependiendo del tipo de viaje- y llegando al destino seleccionado. Por lo que se efectúa un señalamiento en cuanto al lenguaje a utilizar que se relaciona con las diferencias planteadas entre los verbos «conocer» $\mathrm{y}$ «estar» y que no es meramente semántica. Lo cual tiene una significación más profunda, en lugar de decir «conocí París» en realidad debería decirse «estuve en París». El argumento se centra en que sobre los principales atractivos y productos que el viajero visita, ya disponía de información previa, la cual ha recabado o se le ha facilitado por parte de los distintos agentes. En el mismo sentido, y en la mayoría de los casos, frecuentará las zonas más turísticas del lugar, ya definidas previamente. Este planteo implica que hace un uso limitado de la totalidad del espacio, lo que está determinado por tres 
razones principales: 1) el tiempo que estará es breve si se lo compara con su VC; 2) las motivaciones de su viaje; y 3) por la influencia de las distintas promociones/ publicidades que el destino emite.

\section{Grado de involucramiento de La VC en el turismo}

Como se ha señalado anteriormente, el grado de involucramiento tiene dos componentes centrales: 1) el factor humano, donde adquiere un valor significativo el relato de historias asociadas a particularidades de algunos territorios como se puede apreciar en las presentaciones de artesanos de familia de algunas casas de la realeza europea, exprostitutas de Ámsterdam, artistas urbanos, maestros de oficios extintos, entre otros (diario El Cronista, 2019); y 2) el entorno, en este caso se podría mencionar como ejemplo un recorrido de senderismo o un viaje en lancha por un lugar caracterizado por su biodiversidad de flora, fauna, geografía, entre otras.

La atención a la forma de VC (cualquiera sea su origen espacial) puede presentarse en diferentes grados, desde "renunciar», pasando por un "recurrir» a aspectos parciales, hasta el «refleja», donde se la presenta en mayor medida y/o profundidad. A ello se suma de forma transversal dos perspectivas más en cuanto a la evolución de las características de la VC por y/o para el turismo que son: recuerda y renueva. La siguiente tabla intenta dar cuenta de ello en forma esquemática.

CUADRO III. RELACIÓN VC/TURISMO.
5R: RENUNCIA, RECURRE, REFLEJA, RECUERDA, RENUEVA

Fuente: elaboración propia. 


\section{CONSIDERACIONES FINALES}

En el desarrollo de este breve ensayo, se ha intentado resaltar la dimensión espaciotemporal de la relación existente entre la VC y turismo, la cual puede ser analizada desde distintas perspectivas.

Si bien el abordaje de la VC es un elemento recurrente en los estudios turísticos, suele ser analizada desde la perspectiva del turista dejando de lado las características que tienen en otros actores (comunidades, trabajadores, empresarios y gobiernos). El ámbito de la vida cotidiana posibilita realizar una lectura desde el lugar donde se materializan no solo las decisiones del viajero sino también aquellas que, por ejemplo, hacen las políticas turísticas.

Se puede apreciar que existen dos ejes que permiten comprender parte de las acciones. Ellos se corresponden con el relacionamiento con el entorno y el relacionamiento con las personas. Ambos tienen un nivel de análisis de diferente escala, desde un colectivo en su conjunto hasta la diversidad existente en cada uno de los territorios y/o de los individuos.

Las características actuales de las sociedades muestran que los viajes son cada vez más frecuentes o fraccionados en el tiempo lo que atraviesa de manera permanente y constante a la $\mathrm{VC}$ de todos los actores (turistas, comunidades, gobiernos, empresarios y trabajadores).

Si bien espacio y tiempo son dos ejes que pueden ser analizados de forma separada en el campo del turismo, se resalta la importancia del abordaje en forma conjunta, donde no solo se estudie al sujeto (turista) sino que se incorpore al resto de los actores del sistema turístico.

En cuanto a la relación VC y turismo se podría clasificar en cinco modalidades las cuales han sido nominadas como las " $5 \mathrm{R}$ " (renuncia, recurre, refleja, recuerda, renueva). De las cuales las dos últimas son transversales. Categorías que no son excluyentes de otras integraciones cuando se pretende con ellas seguir avanzando en el estudio de cuestiones que aporten a la conceptualización del turismo como un campo interdisciplinario que posibilitara una visión más integral de las prácticas turísticas en lo referente al tiempo y al espacio. 


\section{BIBLIOGRAFÍA}

Berdoulay, V. (2012). «El sujeto, el lugar y la mediación del imaginario», en Lindón, A. y Hiernaux, D. (eds.) (2012). Geografías de lo imaginario. Anthropos Editorial.

Campodónico, R. y Chalar, L. (2014). «Matriz Científica en Turismo: una propuesta integradora». ABET, Juiz De FORA, 4(3), 9-17. https://periodicos.ufjf.br/index.php/abet/article/ view/3056.

Campodónico, R. y Chalar, L. (2019). «Reflexión teórico metodológica sobre el alejamiento/ acercamiento entre vida cotidiana y turismo». Estudios y Perspectivas del Turismo, 28(4), 1087-1113. https://www.estudiosenturismo.com.ar/PDF/V28/N04/v28n4a12.pdf.

Chalar L. (2020). Abordaje de la competitividad del destino turistico desde la articulación productiva intersectorial local. El caso de los sectores turismo y horticultura del Departamento de Salto (Uruguay). Tesis Maestría en Desarrollo y Gestión del Turismo. Universidad Nacional de Quilmes.

Collado Medina, L.M., Medina Cuevas, L.M., Herrera Márquez, A.H. y Moreno ZaGalm, M. (2013). «Innovación curricular en turismo: un campo de estudio y referentes para la formación universitaria». Actualidades Investigativas en Educación, 13(1), 1-39. https:// doi.org/10.15517/aie.v13i1.11708.

Diario El Cronista (2 de septiembre de 2019). Para el gurú de los hoteles 5 estrellas si un huésped se siente como en su casa hice todo mal. https://www.cronista.com/clase/dixit/Parael-guru-de-los-hoteles-5-estrellas-si-un-huesped-se-siente-como-en-su-casa-hice-todomal-20190902-0003.html.

Diario El País (6 de septiembre de 2019). Hoteles uruguayos que agudizan el ingenio para paliar la caída de huéspedes. https://www.elpais.com.uy/el-empresario/hoteles-uruguayos-agudizan-ingenio-paliar-caida-huespedes.html.

Gerlero, J.C. y Taranda, D. (2005). «El Turismo: una perspectiva de análisis desde la vida cotidiana», 133-142, en Condet (2005), Realidad, Enigmas y Soluciones en Turismo, Universidad Nacional del Comahue. http://www.condet.edu.ar/cndt/images/articulos/Articulo\%20 9\%20Gerlero\%20Taranda\%20Condet\%204.pdf.

Giddens, A. (1984). «La constitución de la sociedad: bases para la teoría de la estructuración», Amorrortu Editores, en Hall, C.M. (2009). El turismo como ciencia social de la movilidad. Editorial Síntesis.

Hall, C.M. (2009). El turismo como ciencia social de la movilidad. Editorial Síntesis.

Hannam, K., Sheller, M. y Urry, J. (2006). «Editorial: Mobilities, immobilities and moorings». Mobilities 1(1), 1-22, en Onghena, Y. y Milano, C. (2015). Certezas e incertezas del turismo: movilidad y encuentro. Notes internacionals CIDOB 116, Universitat Autónoma de Barcelona. https://www.cidob.org/es/publicaciones/serie_de_publicacion/notes_internacionals/ n1_116_certezas_e_incertezas_del_turismo_movilidad_y_encuentro/certezas_e_incertezas_del_turismo_movilidad_y_encuentro.

Heller, A. (1998). La revolución de la vida cotidiana. Ediciones Península.

Koselleck, R. (1993). Futuro Pasado. Para una semántica de los tiempos históricos. Ediciones Paidós.

Lindón, A. (2012). «¿Geografías de lo imaginario o la dimensión imaginaria de las geografías del Lebenswelt?», en Lindón, A. y Hiernaux, D. (eds.) (2012). Geografias de lo imaginario. Anthropos Editorial. 
Lindón, A. y Hiernaux, D. (eds.) (2012). Geografias de lo imaginario. Anthropos Editorial.

Mantecón, A. (2008). La experiencia del turismo: un estudio sociológico sobre el proceso turístico-residencial. Icaria Editorial.

Pichón Rivière, E. y Pampliega, A. (1985). Psicología de la vida cotidiana. Ediciones Nueva Visión.

Santos, M. (1994). Técnica do Tempo Espacial Globalização e meio técnico-científico informativo. Hucitec.

Unwin, T. (2000). «A waste of space? Towards a critique of the social production of space...». Trasactions of the Institute of British Geographers, MS, 25(1) 11-29. https://doi.org/10.1111/j.00202754.2000.00011.x.

Urry, J. (2004). La mirada del turista. Universidad San Martín de Porres, Lima. https://doi. org/10.24265/turpatrim.2001.n3.03. 
